\title{
Improving the performance of the single-dish Cherenkov telescope MAGIC through the use of signal timing
}

E. Aliu ${ }^{a}$, H. Anderhub ${ }^{b}$, L.A. Antonelli ${ }^{c}$, P. Antoranz ${ }^{\text {d }}$, M. Backes ${ }^{\text {e }}$, C. Baixeras ${ }^{f}$, J.A. Barrio ${ }^{d}$, H. Bartko $^{g}$, D. Bastieri ${ }^{\text {h }}$, J.K. Becker ${ }^{\mathrm{e}}$, W. Bednarek ${ }^{\mathrm{i}}$, K. Berger ${ }^{\mathrm{j}}$, E. Bernardini ${ }^{\mathrm{k}}$, A. Biland ${ }^{\text {b }}$, R.K. Bock ${ }^{\text {g,h }}{ }^{\text {, G. Bonnoli }}{ }^{\mathrm{l}}$, P. Bordas ${ }^{\mathrm{m}}$, D. Borla Tridon ${ }^{\mathrm{g}}$, V. Bosch-Ramon ${ }^{\mathrm{m}}$, T. Bretz ${ }^{\mathrm{j}}$, I. Britvitch ${ }^{\mathrm{b}}$, M. Camara ${ }^{\mathrm{d}}$, E. Carmona ${ }^{\mathrm{g}}$, A. Chilingarian ${ }^{n}$, S. Commichau ${ }^{\text {b }}$, J.L. Contreras ${ }^{d}$, J. Cortina ${ }^{\text {a }}$, M.T. Costado ${ }^{\text {o,p }}$, S. Covino ${ }^{c}$, V. Curtef ${ }^{\text {e }}$, F. Dazzi ${ }^{\mathrm{h}}$, A. De Angelis ${ }^{\mathrm{q}}$, E. De Cea del Pozo ${ }^{\mathrm{r}}$, R. de los Reyes ${ }^{\mathrm{d}}$, B. De Lotto ${ }^{\mathrm{q}}$, M. De Maria ${ }^{\mathrm{q}}$, F. De Sabata ${ }^{\mathrm{q}}$, C. Delgado Mendez ${ }^{\circ}, A$. Dominguez ${ }^{\mathrm{s}}$, D. Dorner ${ }^{\mathrm{j}}$, M. Doro ${ }^{\mathrm{h}}, \mathrm{D}_{\text {. Elsässer }}^{\mathrm{j}}$, M. Errando $^{\mathrm{a}}$, M. Fagiolini $^{\mathrm{l}}$, D. Ferenc ${ }^{t}$, E. Fernández ${ }^{a}$, R. Firpo ${ }^{a}$, M.V. Fonseca ${ }^{\text {d }}$, L. Font ${ }^{f}$, N. Galante ${ }^{g}$, R.J. García López ${ }^{\text {o,p }}$, M. Garczarczyk ${ }^{g}$, M. Gaug ${ }^{o}$, F. Goebel ${ }^{g}$, D. Hadasch ${ }^{\mathrm{e}}$, M. Hayashida ${ }^{\mathrm{g}}$, A. Herrero ${ }^{\text {opp }}$, D. Höhne ${ }^{\mathrm{j}}$, J. Hose ${ }^{\mathrm{g}}$, C.C. Hsu $^{g}$, S. Huber ${ }^{j}$, T. Jogler ${ }^{g}$, D. Kranich ${ }^{b}$, A. La Barbera ${ }^{c}$, A. Laille ${ }^{t}$, E. Leonardo ${ }^{1}$, E. Lindfors ${ }^{\mathrm{u}}$, S. Lombardi ${ }^{\text {h }}$, F. Longo q , M. López ${ }^{\text {h }}$, E. Lorenz ${ }^{\text {b,g }}$, P. Majumdar ${ }^{\mathrm{k}}$, G. Maneva ${ }^{\mathrm{v}}$, N. Mankuzhiyil $^{\mathrm{q}}$, K. Mannheim ${ }^{\mathrm{j}}$, L. Maraschi ${ }^{\mathrm{c}}$, M. Mariotti ${ }^{\mathrm{h}}$, M. Martínez ${ }^{\mathrm{a}}$, D. Mazin ${ }^{\mathrm{a}}$, M. Meucci ${ }^{1}$, M. Meyer ${ }^{\mathrm{j}}$, J.M. Miranda $^{\mathrm{d}}$, R. Mirzoyan ${ }^{\mathrm{g}}$, M. Moles ${ }^{\mathrm{s}}$, A. Moralejo ${ }^{\mathrm{a}, *}$, D. Nieto ${ }^{\mathrm{d}}$, K. Nilsson ${ }^{\mathrm{u}}$, J. Ninkovic ${ }^{\mathrm{g}}$, N. Otte ${ }^{\mathrm{g}, \mathrm{w}, 1}{ }$ I. Oya $^{\mathrm{d}}$, R. Paoletti ${ }^{1}$, J.M. Paredes ${ }^{\mathrm{m}}$, M. Pasanen ${ }^{\mathrm{u}}$, D. Pascoli ${ }^{\text {h }}$, F. Pauss ${ }^{\mathrm{b}}$, R.G. Pegna ${ }^{\text {, }}$, M.A. Perez-Torres ${ }^{\mathrm{s}}$, M. Persic ${ }^{\mathrm{q}, \mathrm{x}}$,

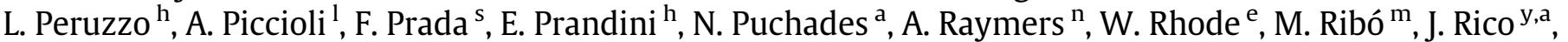
M. Rissi ${ }^{\text {b }}$, A. Robert ${ }^{f}$, S. Rügamer ${ }^{j}$, A. Saggion ${ }^{\text {h }}$, T.Y. Saito ${ }^{g}$, M. Salvati ${ }^{c}$, M. Sanchez-Conde ${ }^{\text {s }}$, P. Sartori ${ }^{\text {h }}$,

K. Satalecka ${ }^{k}$, V. Scalzotto ${ }^{\text {, }}$ V. Scapin ${ }^{\mathrm{q}}$, T. Schweizer ${ }^{\mathrm{g}}$, M. Shayduk ${ }^{g}$, K. Shinozaki ${ }^{\mathrm{g}}$, S.N. Shore ${ }^{\mathrm{z}}$, N. Sidro ${ }^{\mathrm{a}}$, A. Sierpowska-Bartosik ${ }^{r}$, A. Sillanpää ${ }^{\text {u }}$, J. Sitarek ${ }^{g}$, D. Sobczynska ${ }^{\text {i }}$, F. Spanier ${ }^{j}$, A. Stamerra ${ }^{\text {, L.S. Stark }}{ }^{\text {, }}$, L. Takalo ${ }^{\mathrm{u}}$, F. Tavecchio ${ }^{\mathrm{c}}$, P. Temnikov ${ }^{\mathrm{v}}$, D. Tescaro ${ }^{\mathrm{a}, *}$, M. Teshima ${ }^{\mathrm{g}}$, M. Tluczykont ${ }^{\mathrm{k}}$, D.F. Torres $^{\mathrm{y}, \mathrm{r}}$,

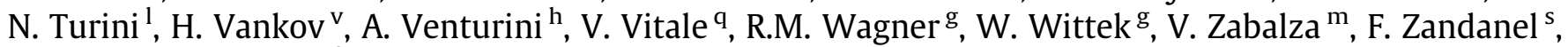
R. Zanin ${ }^{\text {, }}$, J. Zapatero ${ }^{\mathrm{f}}$

\footnotetext{
a IFAE, Edifici Cn., Campus UAB, E-08193 Bellaterra, Spain

${ }^{\mathrm{b}}$ ETH Zurich, $\mathrm{CH}-8093$, Switzerland

${ }^{\mathrm{c}}$ INAF National Institute for Astrophysics, I-00136 Rome, Italy

${ }^{\mathrm{d}}$ Universidad Complutense, E-28040 Madrid, Spain

e Technische Universität Dortmund, D-44221 Dortmund, Germany

${ }^{\mathrm{f}}$ Universitat Autònoma de Barcelona, E-08193 Bellaterra, Spain

${ }^{\mathrm{g}}$ Max-Planck-Institut für Physik, D-80805 München, Germany

${ }^{\mathrm{h}}$ Università di Padova and INFN, I-35131 Padova, Italy

${ }^{\mathrm{i}}$ University of Łódź, PL-90236 Lodz, Poland

j Universität Würzburg, D-97074 Würzburg, Germany

${ }^{k}$ DESY Deutsches Elektr.-Synchrotron, D-15738 Zeuthen, Germany

${ }^{1}$ Università di Siena and INFN Pisa, I-53100 Siena, Italy

${ }^{\mathrm{m}}$ Universitat de Barcelona (ICC/IEEC), E-08028 Barcelona, Spain

${ }^{n}$ Yerevan Physics Institute, AM-375036 Yerevan, Armenia

${ }^{\circ}$ Inst. de Astrofisica de Canarias, E-38200 La Laguna, Tenerife, Spain

${ }^{\mathrm{p}}$ Depto. de Astrofisica, Universidad, E-38206 La Laguna, Tenerife, Spain

${ }^{\mathrm{q}}$ Università di Udine and INFN Trieste, I-33100 Udine, Italy

${ }^{\mathrm{r}}$ Institut de Cienciès de l'Espai (IEEC-CSIC), E-08193 Bellaterra, Spain

${ }^{s}$ Inst. de Astrofisica de Andalucia (CSIC), E-18080 Granada, Spain

${ }^{\mathrm{t}}$ University of California, Davis, CA 95616-8677, USA

u Tuorla Observatory, Turku University, FI-21500 Piikkiö, Finland

${ }^{v}$ Inst. for Nucl. Research and Nucl. Energy, BG-1784 Sofia, Bulgaria

${ }^{\mathrm{w}}$ Humboldt-Universität zu Berlin, D-12489 Berlin, Germany

${ }^{\mathrm{x}}$ INAF/Osservatorio Astronomico and INFN, I-34143 Trieste, Italy
}

\footnotetext{
* Corresponding authors. Tel.: +34 935812839 (D. Tescaro).

E-mail addresses: moralejo@ifae.es (A. Moralejo), tescaro@ifae.es (D. Tescaro).

${ }^{1}$ Present address: UC Santa Cruz, CA-95064, USA.
} 
${ }^{\mathrm{y}}$ ICREA, E-08010 Barcelona, Spain

${ }^{\mathrm{z}}$ Università di Pisa and INFN Pisa, I-56126 Pisa, Italy

\section{A R T I C L E I N F O}

\section{Article history:}

Received 15 August 2008

Received in revised form 6 October 2008

Accepted 14 October 2008

Available online 31 October 2008

\section{Keywords:}

Gamma-ray astronomy

IACT

Cherenkov images

Timing analysis

\begin{abstract}
A B S T R A C T
The Cherenkov light flashes produced by extensive air showers are very short in time. A high bandwidth and fast digitizing readout, therefore, can minimize the influence of the background from the light of the night sky, and improve the performance in Cherenkov telescopes. The time structure of the Cherenkov image can further be used in single-dish Cherenkov telescopes as an additional parameter to reduce the background from unwanted hadronic showers. A description of an analysis method which makes use of the time information and the subsequent improvement on the performance of the MAGIC telescope (especially after the upgrade with an ultra fast 2 GSamples/s digitization system in February 2007) will be presented. The use of timing information in the analysis of the new MAGIC data reduces the background by a factor two, which in turn results in an enhancement of about a factor 1.4 of the flux sensitivity to point-like sources, as tested on observations of the Crab Nebula.
\end{abstract}

(c) 2008 Elsevier B.V. All rights reserved.

\section{Introduction}

Imaging atmospheric Cherenkov telescopes (IACTs) collect the Cherenkov light from extensive air showers (EAS) to form an image. The morphology of the shower image [1] is used to recognize the few $\gamma$-ray initiated showers among the much more numerous hadronic showers initiated by cosmic ray nuclei. This standard approach only exploits the knowledge of the spatial distribution of the Cherenkov photons in the camera plane, but further informations regarding the shower development are in principle available in the photon arrival times [2]. The possibility of using effectively the timing information to improve the performance of IACTs has been explored in earlier works. The HEGRA collaboration measured on their data a time gradient along the major axis in the Cherenkov images [3]. They suggested that this information may be useful to estimate the distance to the shower core and the shower direction in the case of a single Cherenkov telescope, but of limited use in an array of IACTs, where a stereoscopic view of the shower is available. A recent MC study [4] suggests that the use of the time profile of Cherenkov images may lead to important background rejection improvements in future Cherenkov instruments (even if, according to [5], pioneering tests on real data led only to marginal improvements). A different approach to exploit the time information is proposed by the authors of Ref. [6], capitalizing on the different characteristic time spread of the images of gamma-initiated air showers as compared to hadronic showers or images from distant single muons.

The MAGIC (Major Atmospheric Gamma Imaging Cherenkov) telescope is a single-dish Cherenkov telescope, designed for the detection of VHE gamma rays in the $\sim 50 \mathrm{GeV}$ to $\sim 10 \mathrm{TeV}$ band [7]. Its camera is composed of 577 pixels equipped with high quantum efficiency photomultiplier tubes (PMTs). In the first years of operation of MAGIC, the PMT signals were digitized with 300 MSamples/s flash analogic to digital converters (FADCs). In February 2007 the data acquisition of the MAGIC telescope was upgraded with ultra-fast FADCs capable to digitize at 2 GSamples/s $[8,9]$. The implementation of a faster readout might lead to an improvement in the telescope performance for two reasons: a reduction in the amount of NSB (night sky background) light integrated with the real signal, and an improvement in the reconstruction of the timing characteristics of the recorded images. The main aim of this work is to establish whether the timing information is useful in the analysis of single-dish IACT data. In the following we will present an analysis method which makes use of signal timing, and compare its performance to that of the standard MAGIC analysis used up to now.
The timing analysis proposed here is composed of two different parts. The first is the use of the time information to enhance the efficiency and to lower the threshold of the image cleaning procedure, thanks to the introduction of time constraints. The second is the use of additional time-related image parameters in the algorithms for the suppression of the isotropic background of hadron-initiated showers. Although the possibility of using timing to improve the IACT technique was suggested a long time ago, this is, to our knowledge, the first time in which it has been successfully applied to real data.

\section{Analysis method}

When an atmospheric shower triggers the MAGIC telescope, the information of all camera pixels is stored by the Data AcQuisition (DAQ) system. This information consists mainly of the digitized pulse of the PMT corresponding to each pixel in time slices of $0.5 \mathrm{~ns}$. From the digital information of the pulse it is possible, through the so-called signal extractor routine, to reconstruct the number of photons that arrived at the pixel and their mean arrival time. This can be done in several manners. For the current MAGIC data (with $2 \mathrm{GS} / \mathrm{s}$ sampling), a simple cubic spline is built from the FADC readout, and its integral in a range around the highest peak provides a measure of the charge recorded by the pixel. The arrival time is defined as the position of the rising edge of the pulse at $50 \%$ of the peak value. Before the upgrade of the FADC system, the pulse shape and duration was dictated by the artificial stretching introduced in the electronic chain to ensure that the pulse spanned over several FADC samples (then taken every $3.3 \mathrm{~ns}$ ). For those older data, the digital filter algorithm [10], which makes use of the known pulse shape [11], was used. After calibration, the charge $(Q)$ is converted to photoelectrons units (phe). Details about the calibration can be found in [12].

\subsection{Image cleaning}

The information from the pixels is first used to perform the image cleaning, that aims at identifying which pixels belong to the shower image. In Fig. 1 an example of an event before and after the cleaning is shown.

In the MAGIC analysis and reconstruction software (MARS [13]), different cleaning methods can be chosen by the user. The most commonly used is the standard-absolute method. The choice may depend on the sky around the source (galactic or extra-galactic) or the prevailing atmospheric conditions. This procedure uses a 

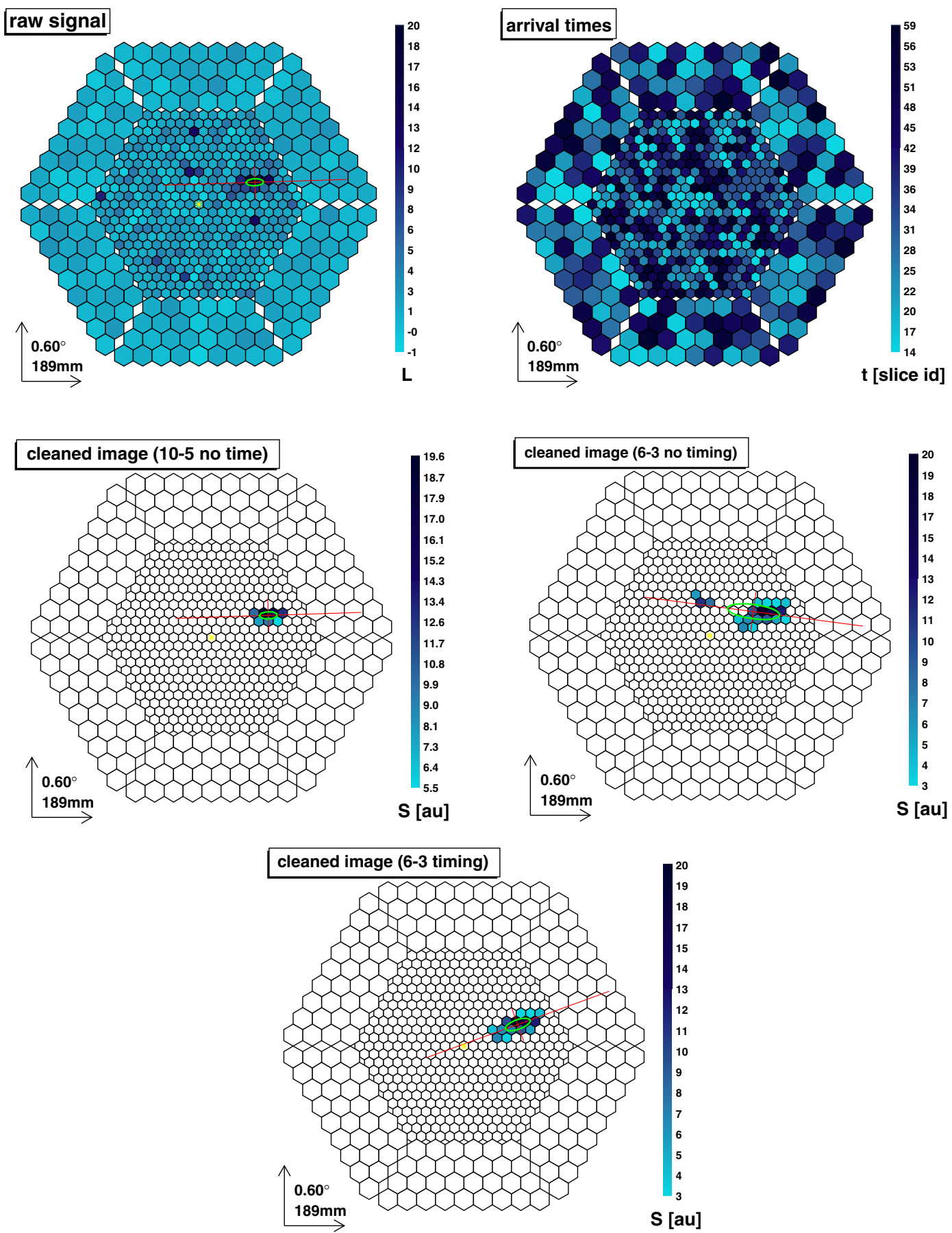

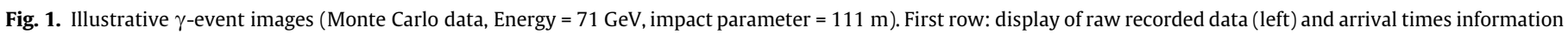

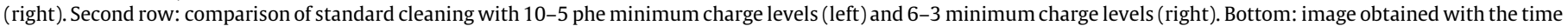
image cleaning (6-3 phe minimum charge levels and $4.5 \mathrm{~ns}$ and $1.5 \mathrm{~ns}$ as time constrains). The simulated gamma-ray source is located in the center of the camera (star).

threshold signal value $q_{1}$ (a fixed value in terms of phe) to select the core pixels, namely all those with charge above $q_{1}$ and which have at least one neighbor fulfilling the same condition. ${ }^{2}$ In a second stage, all pixels which have at least one core neighbor, and whose charge is above $q_{2}$ (with $q_{2}<q_{1}$ ), are included in the image (these are called boundary pixels).

Relaxing the cleaning levels $q_{1}$ and $q_{2}$ results in a larger number of pixels per image, and accordingly a lower analysis energy threshold, since a minimum number of pixels is needed to proceed

\footnotetext{
2 This additional requirement avoids the selection of pixels unrelated to the image whose large charge results from an afterpulse in the PMT.
}

with the analysis. On the other hand, a low cleaning level increases the probability to include in the cleaned image a noise pixel (mainly due to NSB or other unwanted light pollution). The inclusion of pixels unrelated to the shower degrades the image parameters and worsens the performance of the subsequent analysis.

Together with the signal intensity also an arrival time value is assigned by the signal extractor to each pixel. These times can be used to further constrain the selection of core and boundary pixels in the image cleaning algorithm: Cherenkov flashes are very brief (of the order of few ns), and NSB photons produce pulses asynchronous with respect to the pulses of the shower image. A timing coincidence window between the mean arrival time and the single 
pixel arrival time can avoid to confuse NSB signals with real image tails. This further constraint allows to relax the cleaning levels $q_{1}$ and $q_{2}$, lowering in this way the energy threshold. The time information has already been used for the image cleaning in the analysis of the observations of the Crab Nebula with MAGIC [14]. However, the algorithm used in that analysis differs from the one we are proposing here and can be found in detail in [15].

The procedure used in this work can be summarized in this way:

- After selecting the core pixels in the same way as in the standard procedure, we reject those whose arrival time is not within a time $\Delta t_{1}$ of the mean arrival time of all core pixels.

- In the selection of the boundary pixels we add the constraint that the time difference between the boundary pixel candidate and its neighbor core pixels is smaller than a second fixed time constraint $\Delta t_{2}$.

The charge levels of the standard cleaning commonly used in the past in the analysis of MAGIC data are $q_{1}=10$ phe for the core pixels and $q_{2}=5$ phe for the boundary pixels. For the time-cleaning approach, the charge threshold levels were decreased to 6 and 3 phe, respectively. Concerning the time constraints, the values $\Delta t_{1}=4.5 \mathrm{~ns}$ and $\Delta t_{2}=1.5 \mathrm{~ns}$ were selected. The choice of these values is supported by a study based on Monte Carlo data (see $[16,17]$ for more details), in which we have assumed "dark night" conditions (and hence the used criteria would not be optimal during moon light or twilight observations when the number of noise photons is higher). The setting of these time constrains resulted also not very critical for choices within $\simeq 1$ ns respect to the values used here.

In Fig. 1 an example event is shown. The image footprint is visible in the arrival time display (upper right plot) because of the short duration of the Cherenkov flash, illustrating the validity of the time image cleaning approach. The arrival times of the signal pixels are distributed within few ns. The other pixels have, as expected, a random arrival time distribution. In the second and third rows of Fig. 1 the same event is plotted after applying different cleaning methods.

\subsection{Timing characteristics of the shower images}

As previously introduced, Cherenkov images present some timing features, the most important of which is a dependency between the timing profile along the major axis of the image and the impact parameter (IP) of the shower. The model proposed in [3] explains well this relationship. In case of a small impact parameter (IP $\leqslant 60 \mathrm{~m}$ ), the light emitted in the higher part of the shower (the shower head) will arrive delayed with respect to the light emitted in the lower part of the shower (the tail), since the photons emitted first travel slower (at a speed $c / n$ ) than the ultra relativistic particles of the shower that produce the photons at lower altitudes. In case of a larger impact parameter (IP $\geqslant 120 \mathrm{~m}$ ), the effect just described is reduced or even inverted, as the arrival time from the tail becomes the sum of the times spent in the paths of particles and photons, respectively. In this latter situation, the photons emitted in the lower part of the shower will arrive later than the photons emitted in the upper part. Events with an intermediate impact parameter show a flat time profile. These features are well visible in the templates of average Monte Carlo gamma-ray images on the MAGIC camera (Fig. 2), created by the superposition of many events at fixed values of energy and impact parameter. These are part of a dedicated MC sample produced for a different study [18] on the applicability of the so-called "model analysis" [19] to the MAGIC data. In these templates, it is possible to recognize the dependency of the timing structure with the IP: the arrival time increases from shower head (bottom part of the images) to shower tail at large impact parameter, and from tail to head for small impact parameters.

\subsection{Definitions of time parameters}

In order to exploit the timing characteristics of the showers in the analysis stage, some time-related image parameters have to be introduced. A linear fit of the arrival time versus the distance along the major image axis provides an easy way to characterize the time profile of a shower image. Another useful quantity may be the overall spread of the arrival times of all pixels surviving the cleaning. Based on these considerations, two new time-related image parameters have been introduced:

- Time Gradient: this parameter measures how fast the arrival time changes along the major image axis. The pixel coordinates are projected onto this axis, reducing the problem to one dimension. Then the arrival time versus the space coordinate along the major axis is fitted to a linear function $t=m \cdot x+q$. The slope $m$ is called in the following Time Gradient of the image. The sign of this parameter is positive if the arrival time increases as we move away from the location of the source on the camera, negative otherwise. It is therefore a parameter which depends on the position of the candidate gamma-ray source.

- Time RMS: the root mean square of the arrival times of all pixels belonging to the image after cleaning. It measures the spread of the arrival times irrespective of the pixel position in the camera. This parameter has been suggested as a possible background discriminator in [6]. It must be noted that due to the time structure of the events, this parameter is correlated with the Time Gradient.

For the geometrical reasons explained in the previous section, the Time Gradient is well correlated with the impact parameter, as can be seen in the left panel of Fig. 3. On the other hand, the clas-
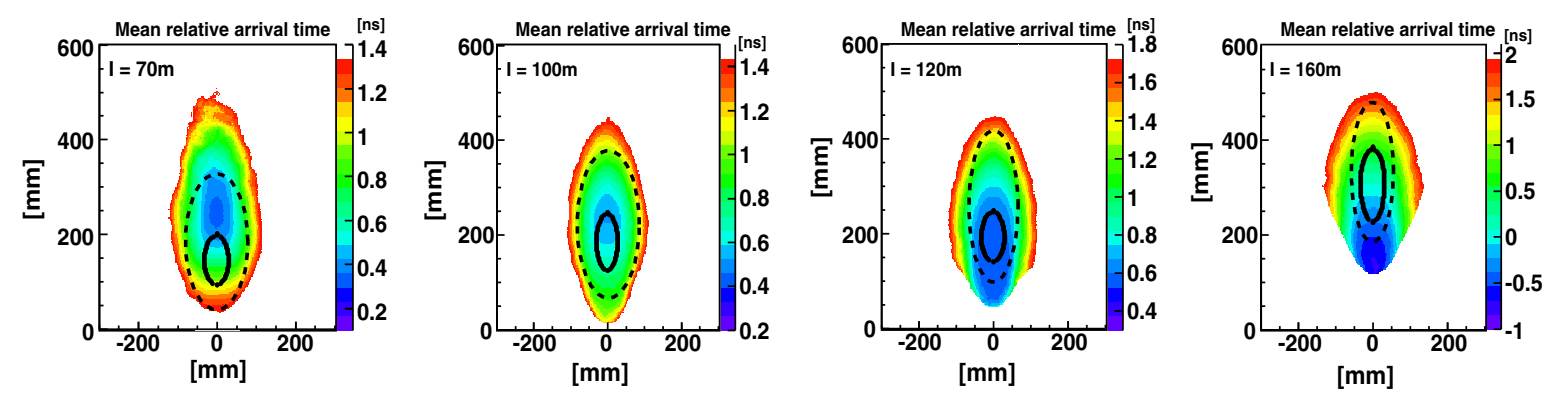

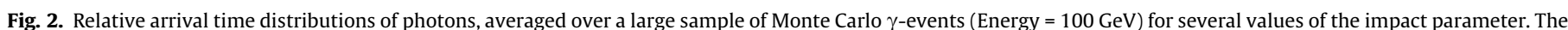

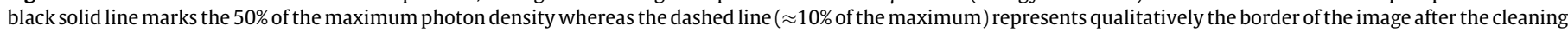
procedure. The time profile of the recorded images changes clearly depending on the IP of the primary shower. The source is located in the $(0,0)$ position. Plots from [18]. 


\section{TIME GRADIENT vS IP}

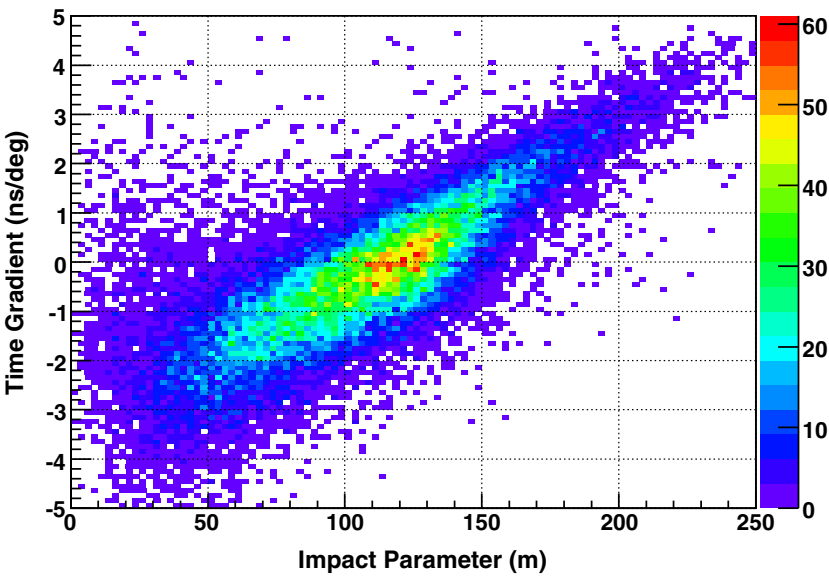

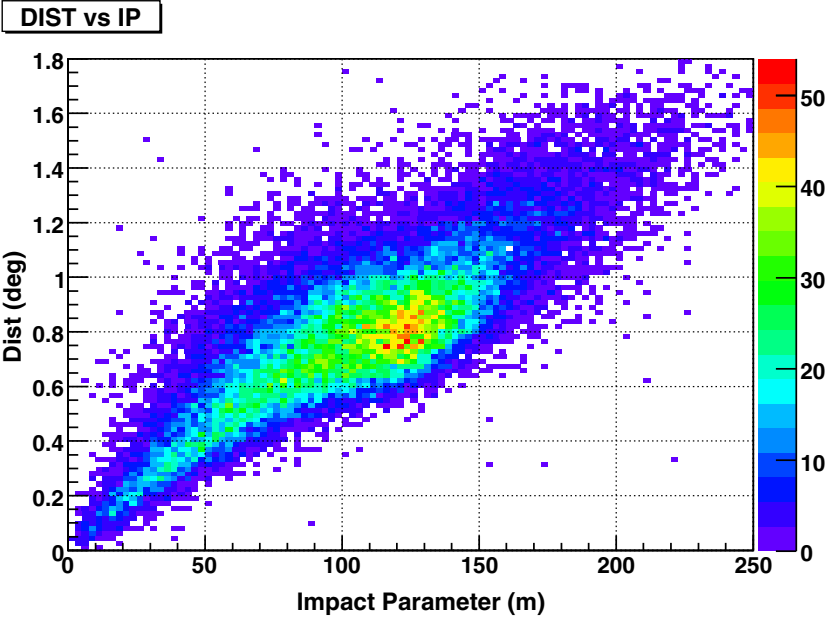

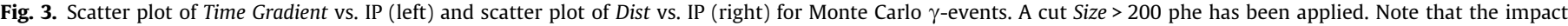

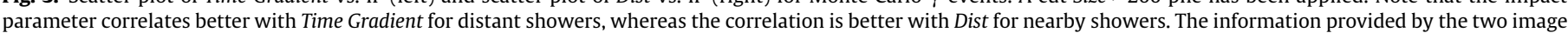
parameters is hence complementary.
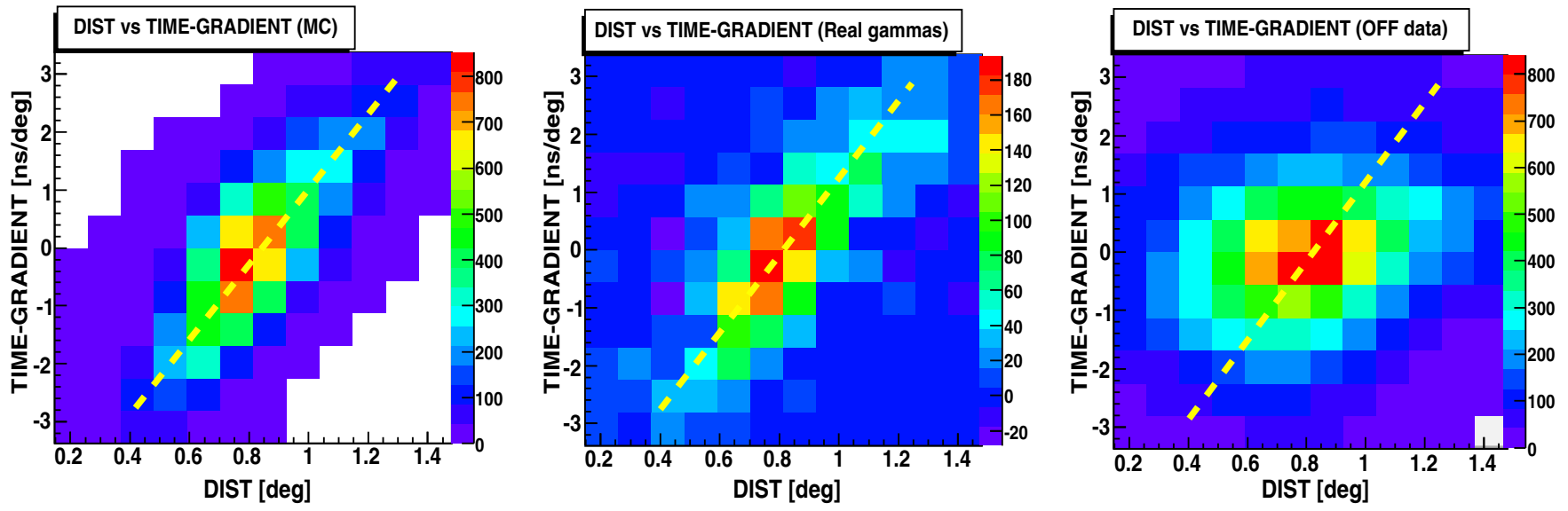

Fig. 4. Time Gradient vs. Dist parameter correlation for MC $\gamma$-rays (left), real $\gamma$-rays (center) and background (right) data.

sical Dist parameter, which is the angular distance from the image center of gravity to the source location on the camera, is also correlated to the impact parameter for gamma-rays coming from a point-like source: as we increase the impact parameter, the image gets longer and moves away from the source, as we observe it at an increasingly larger angle. ${ }^{3}$ A consequence of this is that Time Gradient is correlated with Dist for gamma-ray images from a point-like source (see Fig. 4), whereas no such correlation exists for hadron images, since hadron showers are distributed isotropically, and therefore no strong correlation of Dist and the impact parameter is expected for them. Already from this, one can expect some improvement in the background discrimination through the use of the Time Gradient in the analysis.

Another way of looking at this is the following: the shower direction is not well determined by a single IACT. When observing a point-like source, all gamma-ray images will be pointing (within $\simeq 10^{\circ}$ ) towards the source location on the camera, but so will many background cosmic ray showers whose axes are coplanar with the line pointing from the mirror dish center toward the source. The bare shower shapes allow to eliminate some of those, but the timing profile provides additional independent information to recognize the gamma-rays (the images with "consistent" values of Dist

\footnotetext{
${ }^{3}$ Fluctuations in the shower development make that, even for a fixed energy, the altitude at which it develops changes from event to event, which blurs the correlation of Dist and the impact parameter.
}

and the Time Gradient) and reject the background, and is therefore expected to improve the performance of the analysis.

Note that in stereoscopic IACT systems the determination of the shower direction and the impact parameter is obtained by the intersection of the multiple shower images, and thus the information that could be provided by the timing is redundant. ${ }^{4}$ Therefore, the results obtained in this study should not be extrapolated to the case of stereo observations.

Regarding the Time RMS, it has been suggested in [6] that it may be of help in identifying triggers produced by single, large impact parameter muons (whose images may otherwise be gamma-like), as well as other hadron-initiated showers (since their Time RMS distribution has, with respect to that of gamma-rays, a longer tail towards large values).

\subsection{Role of the Monte Carlo simulation}

Making sure that the Monte Carlo reproduces the features of the real data is very important to perform a good background rejection and energy estimation. In the MAGIC analysis, both tasks rely heavily on the MC simulated events. The MC is also crucial when the gamma-ray flux of a source is computed, since the estimation of

\footnotetext{
${ }^{4}$ In the special case of a two telescopes stereo system, the impact parameter can
} still be poorly determined for some degenerate events. 
the collection area is done using a Monte Carlo "test" sample. Therefore, the detector simulation has been updated to reproduce the digitization features of the new $2 \mathrm{GS} / \mathrm{s}$ digitization system: beyond the higher digitization speed, also the level of electronic noise and the overall precision of the time determination have been adjusted, taking into account the entire electronics chain. The time resolution can be estimated from the calibration events (light pulses of $\sim 2$ ns duration), looking at the distribution of the arrival time difference between any two camera pixels. The RMS of the distribution is $550 \mathrm{ps}$. This correspond to a time resolution for a single pixel of $550 / \sqrt{2}=390$ ps (see [9] for details). Actually, Cherenkov pulses are generally faster than the calibration pulses, and hence, for a pulse of comparable amplitude, showers signals have a better resolution.

A demonstration of the Time Gradient-Dist correlation described in Section 2.3 can be seen in Fig. 4. The left plot of the figure is made with pure $\gamma$-MC events while the central panel displays the difference between on-source and off-source distributions (from a Crab sample described later), and therefore shows the distribution of the gamma-ray excess. A correlation Time Gradient-Dist is present in both cases. Such correlation is almost completely suppressed for hadron images (even after a cut in the Alpha parameter), as shown in the plot on the right.

\section{Experimental results}

MAGIC observations are performed mainly in two modes: onoff and wobble. In the former, the telescope points directly at the source to obtain the on-source data whereas the off data, used to estimate the background, are taken by pointing at a region of the sky where no signal is expected. The wobble mode eliminates the need for taking dedicated off runs [20]. The telescope is not aimed directly at the source, but slightly off ( $0.4^{\circ}$ away). In this way, the source does not occupy a privileged position in the camera, and the background can be estimated by re-doing the analysis with respect to points on the camera ("false-sources") which are expected to be equivalent to the source location (for instance the point symmetric to the source with respect to the camera center). The "wobbling" consists in changing the telescope pointing every 20 min between two symmetric sky directions around the source, which is an additional guarantee of the equivalence of source and false-source against effects like inhomogeneities in the camera response or the dependence of acceptance with the zenith angle. For the studies presented in this paper, the background has always been estimated from one single false-source, located opposite to the source w.r.t. the camera center. The main disadvantage of the wobble method is a small reduction of the trigger efficiency leading to a reduction of $\simeq 15-20 \%$ in the nominal flux sensitivity, since the trigger area is limited to $\simeq 1^{\circ}$ around the camera center.

The data sample chosen for this study consist of $5.7 \mathrm{~h}$ of Crab Nebula observations performed in wobble mode during the nights of the 7th, 9th, 15th and 17th of February 2007 (soon after the installation of the new MUX FADCs readout) at a zenith angles smaller than $30^{\circ}$. Weather conditions were good during all the nights considered.

\subsection{Analysis comparison strategy}

In order to compare the sensitivity with and without the help of the timing information, three different analyses of the above mentioned Crab Nebula data sample were performed:

(1) The standard analysis commonly performed on the MAGIC data before the upgrade of the DAQ. The image cleaning levels were 10 and 5 phe (see Section 2.1), and no time informa- tion was used. The standard image parameters (Size, Width, Length, Dist, Conc and the third moment along the major axis, dubbed $M 3$ long $^{5}$ ) were used to perform the $\gamma /$ h separation. This is the reference analysis for the comparison.

(2) An analysis using 6-3 phe as cleaning levels, with the time constraints described in Section 2.1. The same standard parameters of analysis 1 were used for $\gamma /$ h separation. This analysis is meant to evaluate the effect of the time cleaning.

(3) The same 6-3 phe time cleaning of analysis 2 is used. In this analysis, in addition to the standard image parameters, the Time RMS and the Time Gradient image parameters (see Section 2.3) were used as input for the background rejection. This analysis is meant to evaluate the analysis improvement due to the timing parameters (used together with the time cleaning).

In all cases the image parameters were the input to the Random Forest (RF) event classification algorithm [21], which was used to perform the $\gamma / \mathrm{h}$ separation task. The training samples for the construction of the RF are a MC gamma sample, and a sample of real off data to represent the background. When applied to the data, the RF tags each event with a single value called Hadronness (ranging from 0 to 1 ) which is a measure, based on the image parameters, of the likelihood that the event is a background event.

The sum of the signals (in phe) of the two pixels with highest signal (Size-2) was used as parameter to select event samples of different energies. Like the classical event Size, Size-2 is correlated with energy, but unlike Size, it is very weakly dependent on the cleaning levels. ${ }^{6}$ If we had chosen the total Size to define the samples, we would have faced the problem that they would correspond to different energies in the three analyses, therefore making the interpretation of the results more difficult.

Three different bins of Size-2 are considered in this work: the first one (Size-2 $>100$ phe) corresponds to the energy range where the integral flux sensitivity of MAGIC is best (resulting in a peak gamma energy of around $280 \mathrm{GeV}$ ); the second bin (40 phe <Size- $2<100$ phe) is intended to study the performance at intermediate energies (peak energy $\simeq 150 \mathrm{GeV}$ ). Finally, the performance for gamma-rays below $100 \mathrm{GeV}$, which will be discussed in a separate section, has been evaluated in the Size-2 range from 20 to 40 phe. The estimated energy distributions for the excess events in each of the three Size-2 bins just mentioned (obtained from the real data sample) are shown in Fig. 5.

\subsection{Background rejection}

For the two higher Size-2 bins considered, a series of three Alpha plots are shown in Figs. 6 and 7. The first Alpha plot is relative to the standard analysis (1), the second to the time-cleaning analysis (2) and the third to the time cleaning and time parameters (3). In the case of analysis 1, the Alpha and Hadronness cuts are optimized to obtain the best statistical significance of the excess. For the analyses 2 and 3, the Hadronness cut was chosen so that we got the same number of excess events as in analysis 1 (after applying the same Alpha cut). In this way we can easily compare the background suppression provided by each analysis procedure. Note that the histograms with error bars represent the Alpha distribution of the excess events, instead of the usual plot showing the on-source data

\footnotetext{
${ }^{5}$ This measures the image asymmetry along its major axis. It is a source-dependent parameter, since its sign is referred to the source position on the camera. The sign is defined such that it is positive when the shower head is closer to the source than the shower tail, as is the case for properly reconstructed gamma-rays.

${ }^{6}$ Considering two different image cleanings and applying the same Size-2 cut, the two data samples obtained will contain essentially the same events, differing only in the events that survive just one of the cleanings.
} 

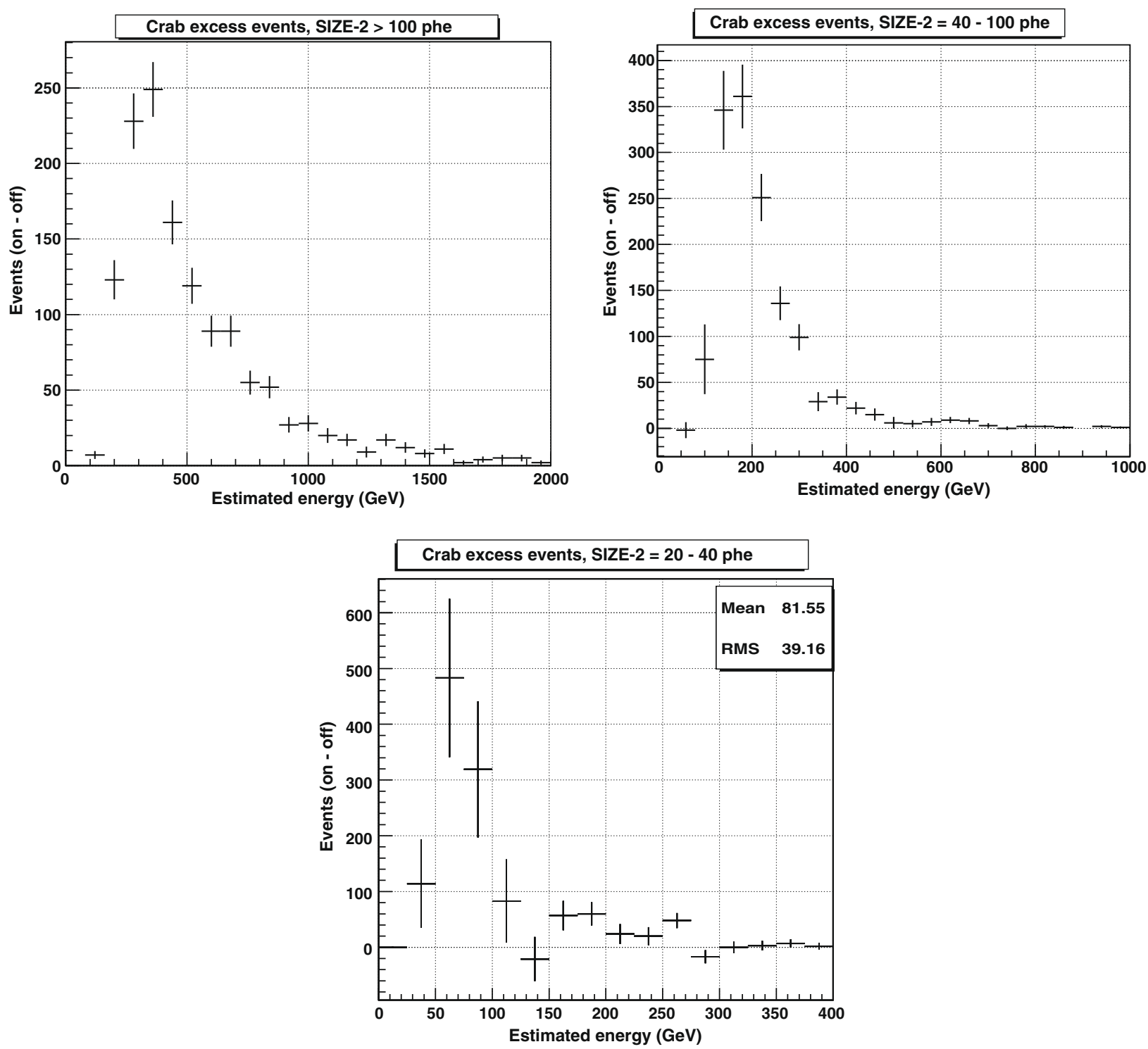

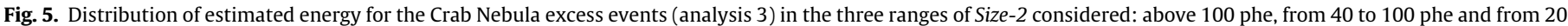
to 40 phe.

before the background subtraction. In this way we can immediately see that the gamma excess is similar in all three analyses, regardless of the background level.

The main result from this comparison is that the use of the time cleaning and the time parameters allows to halve the residual background while keeping the same number of excess events, with respect to the analysis using no time information. This can clearly be seen in Figs. 6 and 7 and the corresponding tables, Tables 1 and 2 . Note that the quoted significance values are calculated using only one false-source position for the background estimation, so the ratio of on-source to off-source exposure is one. The results for the lowest Size-2 bin will be discussed in Section 3.4.

\subsection{Flux sensitivity to point sources}

From the results of the Crab Nebula observations we can estimate the flux sensitivity to point sources achievable with the different analyses. We define the flux sensitivity as the minimum gamma-ray flux detectable in $50 \mathrm{~h}$, where "detectable" means that the excess of gamma-rays corresponds to a signal to noise ratio of five $\left(N_{e x c} / \sqrt{N_{b g}}=5\right)$. This is the standard definition commonly used in the field, but note that it does not correspond exactly to a " $5 \sigma$ detection", because the real significance is usually computed with the Li and Ma formula [22] which takes into account the uncertainty in the determination of the background.

The flux sensitivity depends on the strength of the background discrimination cut (Hadronness $<H_{\max }$ ). Actually, the cuts which maximize the statistical significance of the excess from a strong source like the Crab Nebula, as used in the previous section, are not the ones resulting in the best flux sensitivity: weak sources require tighter cuts. In this section we present the results of a scan of the Hadronness cut values, shown in Fig. 8: the flux sensitivity (in percentage of the flux of the Crab Nebula) is plotted as a function of the rate of excess events. Each Hadronness cut of the scan leads to a different rate of excess and background events and thus to a different flux sensitivity. The figures correspond to the two Size-2 bins considered in the previous section. The black triangles represent the standard analysis 1 , whereas the blue squares and red circles refer to the analysis 2 (with the time cleaning) and 3 (time cleaning and time-related parameters). Note that, since the values are derived from real Crab Nebula observations, the flux percentage is relative to the true Crab flux, and not to the simple power- 

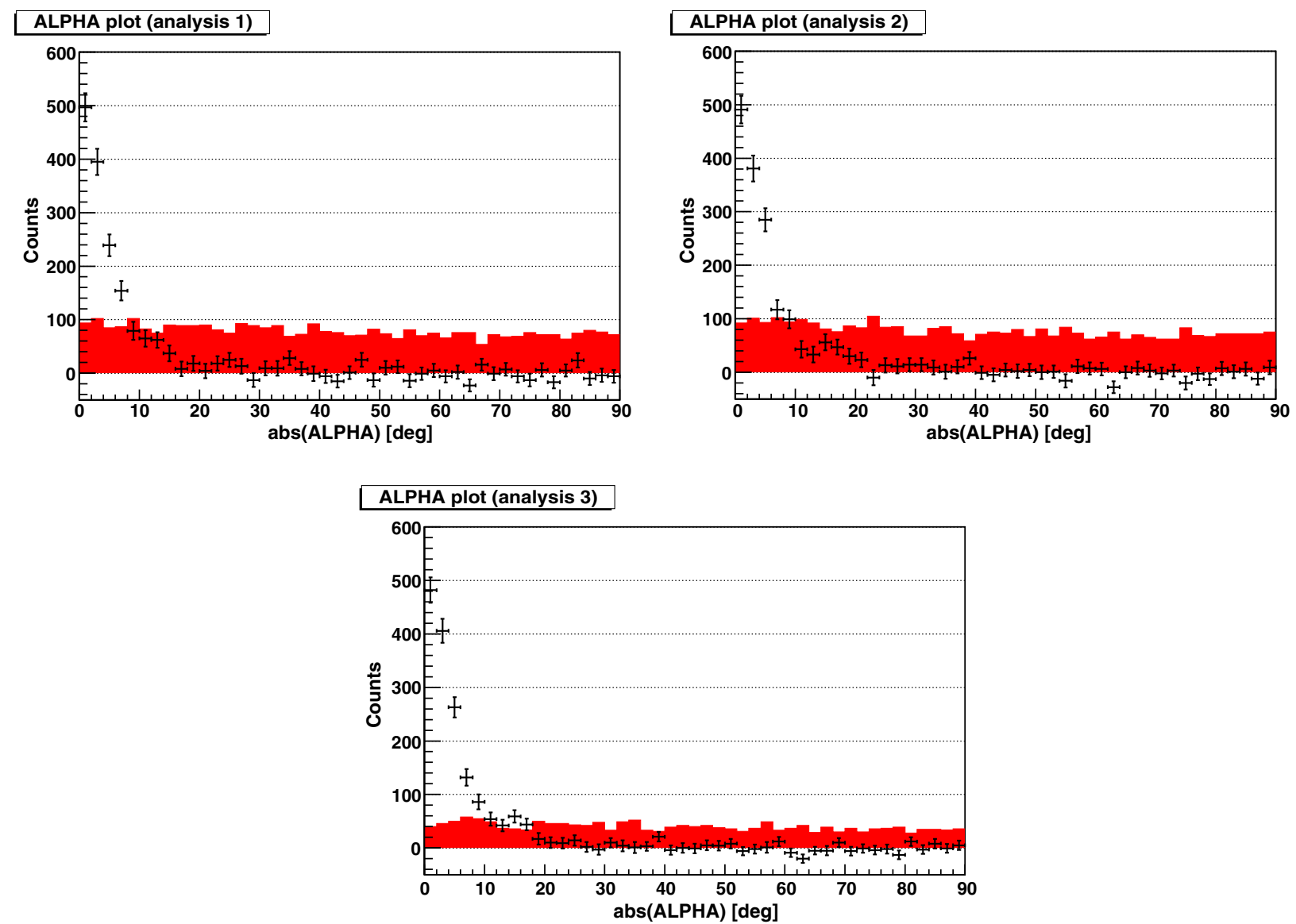

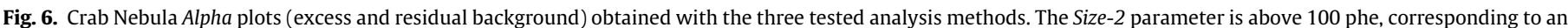

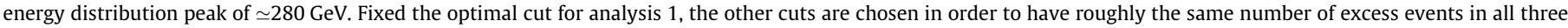
analyses.

law spectrum that is often assumed in sensitivity estimates based on MC. For each choice of Hadronness, a fixed Alpha cut (of $7^{\circ}$ and $10^{\circ}$, respectively) was applied in order to compute the sensitivity. The improvement coming from the use of timing in the analysis is clear in both cases. It must be noted that in the higher energy bin, all of the improvement comes from the use of the timing parameters, whereas in the lower one the introduction of the time cleaning already results in some improvement in sensitivity. The best integral sensitivity that can be reached is around $1.6 \%$ of the Crab flux for a peak energy of $280 \mathrm{GeV}$ (upper panel of Fig. 8).

We have computed also the flux sensitivities in differential bins of estimated energy for analysis 3, shown in Table 3.

\subsection{Use of timing at lower energies}

The background suppression capabilities degrade as we move towards lower energies. This trend can be clearly seen by comparing Figs. 6 and 7 and their corresponding tables: if we focus on analysis 3 , we notice that we move from having a signal nearly seven times larger than the residual background, to having a signal (integrated below the Alpha cut) slightly smaller than the background. This is mainly a result of the worsening of the gamma/hadron discrimination and of the fact that the spectrum of the Crab Nebula is harder than that of the background, although this latter contribution is smaller.

In Fig. 9 we show the results for analyses 2 and 3 in the Size-2 range from 20 to 40 phe, where most of the excess comes from sub-100 GeV gamma-rays (see third pad of Fig. 5). Given the mod- est signal (a mere $5.7 \sigma$ significance in analysis 3), we have in this case adjusted the cuts to obtain the same background rate ( $\simeq 80$ events/min) in both analyses, and then compared the gamma-ray excesses. Once more, the improvement in performance due to the introduction of the timing is clear, though less significant due to the large residual background. With roughly the same background rate the excess rate for analysis 2 is $2.5 \pm 0.7 \gamma / \mathrm{min}$ whereas for analysis 3 it is $4.0 \pm 0.7 \mathrm{\gamma} / \mathrm{min}$. In this energy range, analysis 1 even fails to produce a significant signal, due to the high cleaning levels.

For these low energies, the background overwhelms the signal even of a strong source like Crab, and is actually setting a further limitation for the observation of weak sources. The signal must not only be statistically significant, but also well above the systematic uncertainty in the determination of the background, which is at least of a few percent and unlike statistical significance, it does not get better with longer observation times.

It has to be noted that, even after strong background rejection cuts, a certain amount of "irreducible" background survives. The background rejection power of the imaging technique degrades fast with the lowering of the energy of the primary gamma-ray. The reason of this degradation can be attributed both to the physics of the air showers and to the technical limitations of the IACT instruments. The irreducible background is made up by shower images which are similar to gamma induced images in all of the image parameters used for the event discrimination. This may reflect the shortcomings of the instrument in recording the small and faint images of low energy showers, resulting from its limited 

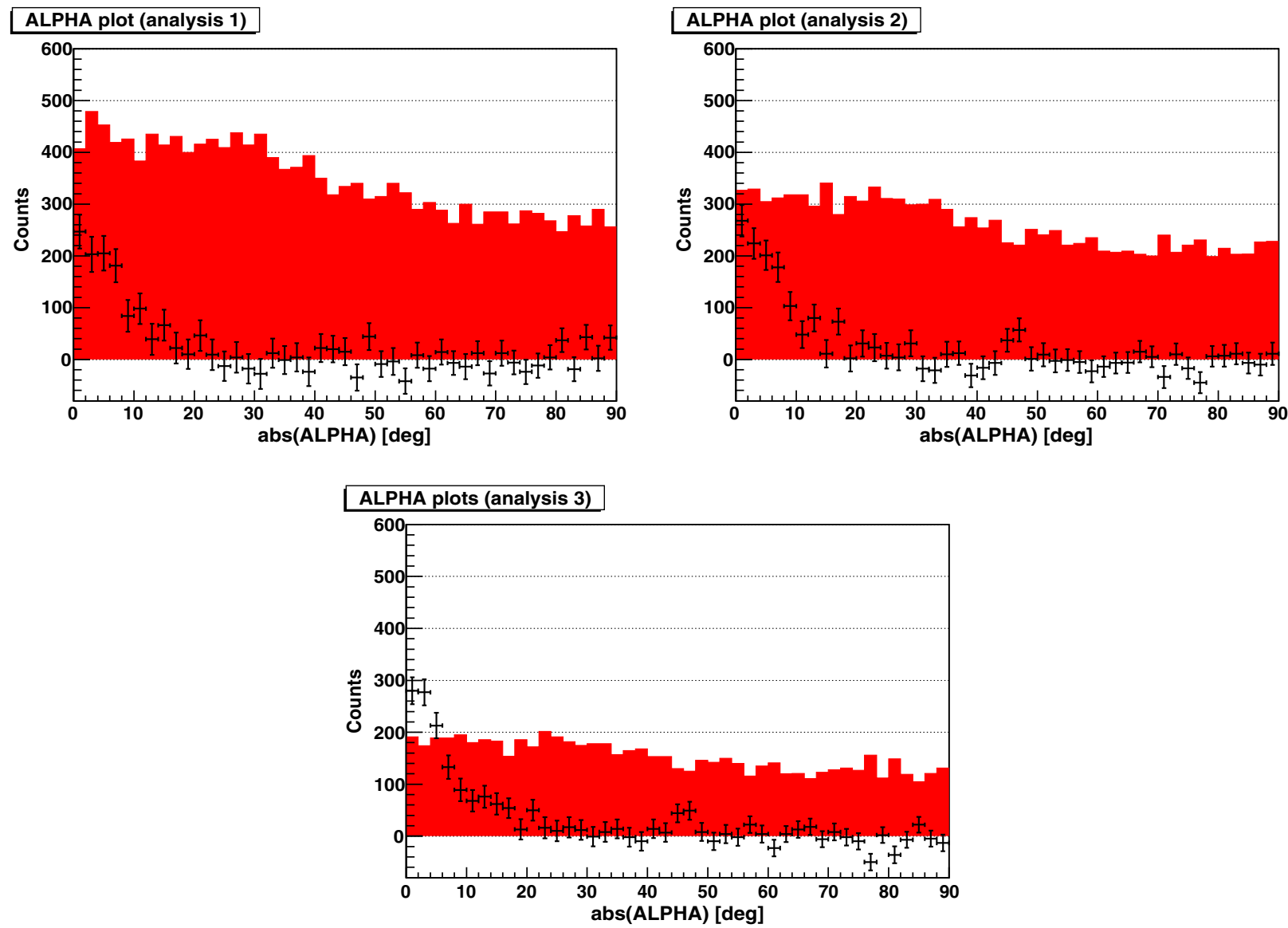

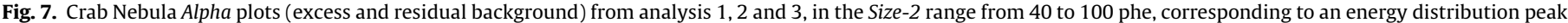
of $\simeq 150 \mathrm{GeV}$.

Table 1

Statistics of the plots in Fig. 6 (Size-2 $>100$ phe; $E_{\text {peak }} \simeq 280 \mathrm{GeV}$ ), obtained with $5.7 \mathrm{~h}$ of observation.

\begin{tabular}{llllll}
\hline Analysis & $\begin{array}{l}\text { HADR. } \\
\text { cut }\end{array}$ & $\begin{array}{l}\text { Alpha cut } \\
(\mathrm{deg})\end{array}$ & $\begin{array}{l}\text { Excess } \\
(\gamma / \mathrm{min})\end{array}$ & $\begin{array}{l}\text { Background } \\
\text { (events/min) }\end{array}$ & $\sigma_{\text {Li\&Ma }} / \sqrt{h}$ \\
\hline 1 & 0.09 & 8 & $3.78 \pm 0.13$ & $1.08 \pm 0.06$ & 12.5 \\
2 & 0.10 & 8 & $3.75 \pm 0.13$ & $1.14 \pm 0.06$ & 12.3 \\
3 & 0.07 & 8 & $3.78 \pm 0.12$ & $0.57 \pm 0.04$ & 14.0 \\
\hline
\end{tabular}

Table 2

Statistics of Fig. 7 ( 40 phe $<$ Size- $2<100$ phe; $E_{\text {peak }} \simeq 150 \mathrm{GeV}$ ), obtained with $5.7 \mathrm{~h}$ of observation.

\begin{tabular}{llllll}
\hline Analysis & $\begin{array}{l}\text { HADR. } \\
\text { cut }\end{array}$ & $\begin{array}{l}\text { Alpha cut } \\
(\mathrm{deg})\end{array}$ & $\begin{array}{l}\text { Excess } \\
(\gamma / \mathrm{min})\end{array}$ & $\begin{array}{l}\text { Background } \\
(\text { events/min })\end{array}$ & $\sigma_{\text {Li\&Ma }} / \sqrt{h}$ \\
\hline 1 & 0.10 & 12 & $3.00 \pm 0.23$ & $7.58 \pm 0.15$ & 5.5 \\
2 & 0.09 & 12 & $3.01 \pm 0.21$ & $5.62 \pm 0.13$ & 6.2 \\
3 & 0.07 & 12 & $3.12 \pm 0.17$ & $3.29 \pm 0.10$ & 7.8 \\
\hline
\end{tabular}

light collection efficiency and camera pixelization. A larger reflecting surface or an increased quantum efficiency camera, together with a finer pixelization, would obviously improve the accuracy of the reconstructed image parameters. On the other hand, even assuming a perfect IACT detector, there is no guarantee that the intrinsic characteristics of the cascades are different enough to permit to distinguish the nature of the primary particles. The study of the characteristics of the gamma-like background (see for example [23] or [24]) heavily rely on the MC simulation packages. The nature of this irreducible background is attributed to $\pi^{0}-\mathrm{s}$, primary electrons and long flying relativistic particles (like $\mu$-s). Proton-induced air showers typically produce pions in the first interaction stage. The charged pions decay into muons, whereas the $\pi^{0}-s$ (most often decaying into two gammas) originate electromagnetic subshowers. If the energy of the primary particle is above a few hundred $\mathrm{GeV}$, the superposition of the sub-showers from different $\pi^{0}-\mathrm{s}$ with diverging trajectories make hadronic showers to appear wider and more "patchy" than gamma-initiated ones. As is well known, this is the feature of hadron-initiated cascades which allows best to suppress them in the analysis of IACT data. However, as the energy of the hadronic primary goes down, the pion multiplicity drops, and the chances that the light of a single electromagnetic subshower dominates the image recorded by an IACT get larger. This means that, regardless of the characteristics of the telescope, the amount of irreducible hadronic background will necessarily increase as we go down in energy.

Also the background images due to distant muons can easily get confused, by a single-dish IACT, with low energy gammas. Those images have generally a small size which make it difficult to recover information from the shower shape. The time spread of pixel signals has been proposed in [6] as a parameter which may be used to suppress such background, but as we will discuss in Section 4, the method does not seem to work efficiently for MAGIC. The improvement in sensitivity in the $<100 \mathrm{GeV}$ energy range is due to the contribution of the Time Gradient parameter.

In summary, even though the overall background discrimination worsens very fast with decreasing energy, the use of the timing parameters (mainly the Time Gradient) has been shown to 

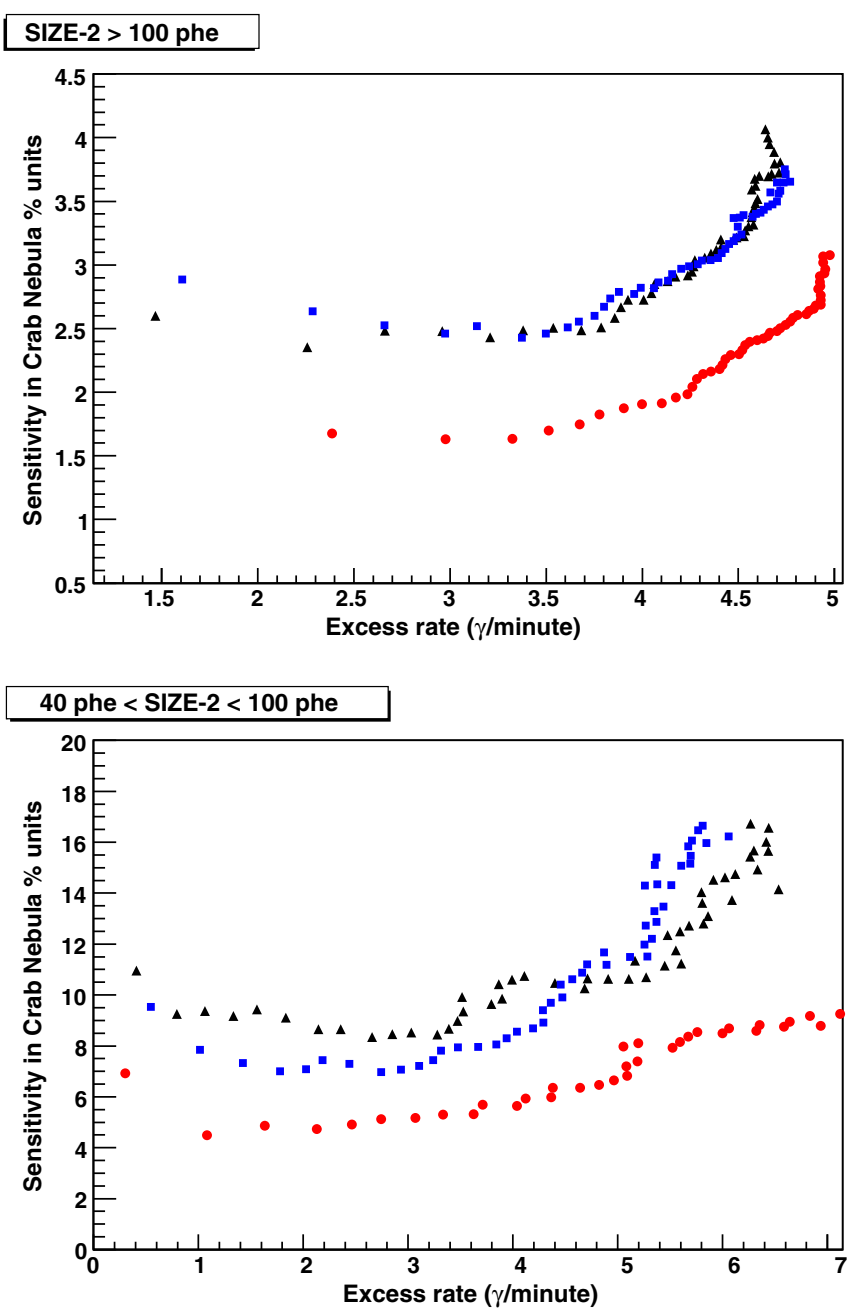

Fig. 8. Sensitivity (for $50 \mathrm{~h}$ ) curves as function of the rate of gamma rays after cuts. The black triangles, blue squares and red circles correspond to analysis 1, 2, and 3, respectively. Upper panel: Size-2 $>100$ phe, corresponding to an energy distribution peak of $\simeq 280 \mathrm{GeV}$. Lower panel: $40<$ Size-2 $(<100$ phe, corresponding to an energy distribution peak of $\simeq 150 \mathrm{GeV}$. The curves are obtained by a scan of the cut in the Hadronness parameter.

improve background supression efficiently in the whole energy range of MAGIC, even below $100 \mathrm{GeV}$.

\subsection{Application of timing analysis to older MAGIC data}

In the previous sections we have discussed exclusively MAGIC data taken after the installation of the fast readout in February 2007. In earlier MAGIC data, the signal sampling was made with FADCs with $300 \mathrm{MSample/s}$. Before digitization, the pulses were stretched up to 6 ns FWHM (10 ns for the low gain), to ensure proper sampling. Timing information was only used in the image cleaning stage of the analysis, to reduce the energy threshold [14]. Only shape parameters were used for background suppression. With that sort of "classical" analysis, no improvement in performance could be seen after the upgrade of the DAQ system: both for the data before and after the upgrade the best integral flux sensitivity achieved in wobble mode was around $2.4 \%$ of the Crab Nebula flux in $50 \mathrm{~h}$. The reduction of the signal integration time (and subsequent reduction of the integrated NSB noise) does not seem to result, by itself, in an improved performance. The reason is that the intrinsic fluctuations of the Cherenkov light recorded by a pixel (coming from the Poissonian photon statistics) dominate over the
Table 3

Sensitivity (\% Crab in $50 \mathrm{~h}$ ) and statistics for some differential energy bins using the time cleaning and the timing parameters in the analysis 3 of Section 3.1. Cuts are optimized separately in each bin with the best sensitivity criteria. Observation time: $5.7 \mathrm{~h}$.

\begin{tabular}{llllll}
\hline $\begin{array}{l}E_{\text {est }} \text { range } \\
(\mathrm{GeV})\end{array}$ & H. cut & $\begin{array}{l}\alpha \text { cut } \\
(\mathrm{deg})\end{array}$ & $\begin{array}{l}\text { Excess } \\
(\gamma / \mathrm{min})\end{array}$ & $\begin{array}{l}\text { Backg. } \\
\text { (events/min) }\end{array}$ & $\begin{array}{l}\text { Sensitivity } \\
(\% \text { Crab })\end{array}$ \\
\hline $100<E<200$ & 0.02 & 12 & $0.70 \pm 0.06$ & $0.23 \pm 0.03$ & 6.3 \\
$200<E<300$ & 0.02 & 8 & $0.80 \pm 0.06$ & $0.11 \pm 0.02$ & 3.7 \\
$300<E<500$ & 0.02 & 8 & $1.00 \pm 0.06$ & $0.09 \pm 0.02$ & 2.8 \\
$500<E<1000$ & 0.04 & 4 & $0.79 \pm 0.05$ & $0.03 \pm 0.01$ & 2.1 \\
$E>1000$ & 0.06 & 4 & $0.28 \pm 0.03$ & $0.005 \pm 0.003$ & 2.1 \\
\hline
\end{tabular}

fluctuations of the NSB light (with a mean rate of about 0.13 phe/ ns in an inner pixel), and therefore the reduction of the NSB noise does not change significantly the precision of the charge measurement in a pixel. ${ }^{7}$

Despite the poorer quality of the timing information recorded in data taken before February 2007, we have also tried to apply the timing parameters defined in Section 2.3 to their analysis. A $8.7 \mathrm{~h}$ Crab data sample taken in good weather conditions during four nights in December 2006 and January 2007, shortly before the change of the DAQ system, was used for this test. It turns out that the improvement in background suppression brought about by the timing parameters on these data is smaller than the one obtained on newer data, shown in Sections 3.2 and 3.3. The best integral sensitivity, achieved for the SIZE-2 $>100$ phe sample, is $1.9 \%$ of the Crab flux.

Although we have not yet been able to investigate this issue in detail, the most obvious reason for the smaller effect of the timing parameters in the analysis of MAGIC data taken before the upgrade of the readout is the worse quality of the timing information. Nevertheless, the precision of the determination of the arrival time of calibration pulses, estimated by studying the reconstructed times for different pixels as discussed in Section 2.4, is roughly the same for both setups ( $\simeq 390$ ps RMS). Calibration flashes make rather large pulses of around 35 photoelectrons per inner pixel [9], and certainly most of the pixels in the processed shower images have smaller signals. On top of that, calibration pulses are wider than pulses from showers ( 4.6 vs. 2.3 ns FWHM). That is, calibration pulses are not representative of the bulk of the pulses which contribute to the images and thus to the time parameters we use in the background discrimination. In conclusion, the study mentioned above, using calibration pulses, is not in contradiction with the naïve expectation of an improvement in the accuracy with which pulse times are reconstructed with the faster sampling. The better performance of timing parameters in the suppression of the background after the FADC upgrade is an indirect evidence that this is the case.

\subsection{Energy estimation}

The Random Forest method can also be used for the estimation of a continuous variable. It is the standard method used in the analysis of MAGIC data for estimating the energy of the showers (under the assumption that they are gamma-rays). In an analogous way as for the background rejection, the RF is trained with MC gammas, whose true energy is known. The main difference is in the way the RF is built: the optimal cut in each node of the trees

\footnotetext{
7 This applies to the "dark night" observations discussed in this paper. For observations under moonlight or in twilight, with a higher rate of NSB photons, the shorter integration time is indeed an advantage in the reconstruction of small showers.
} 

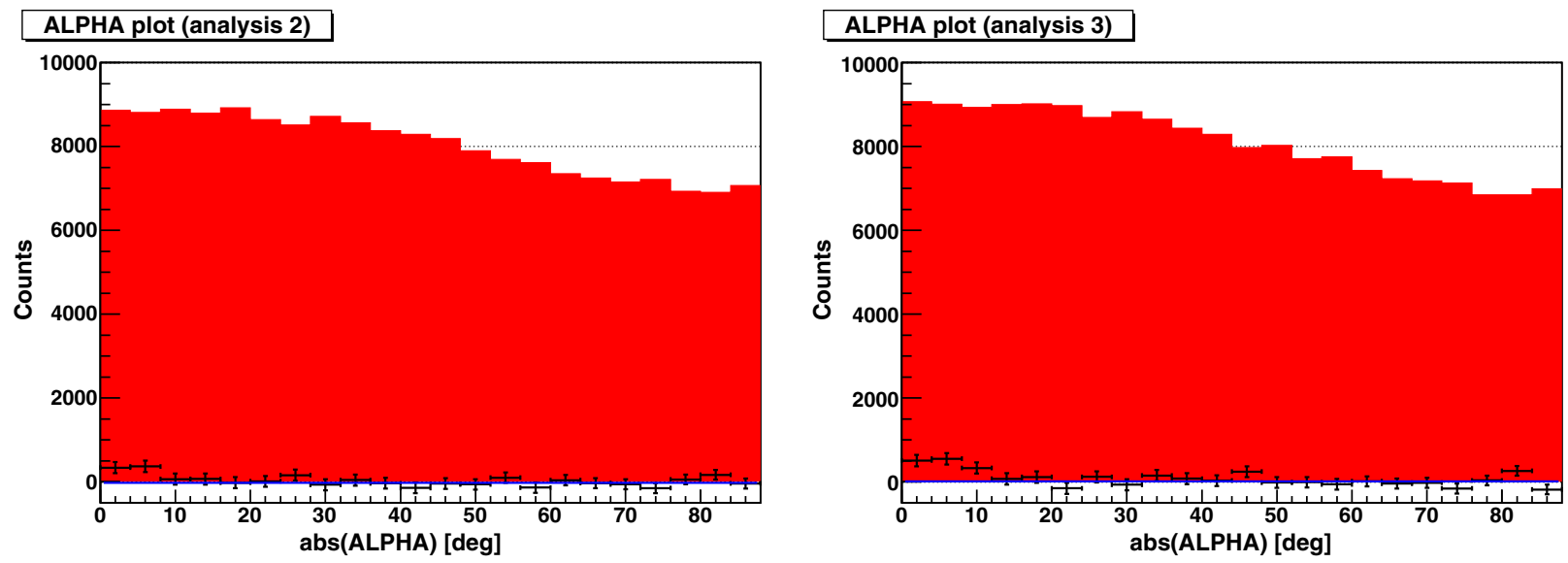

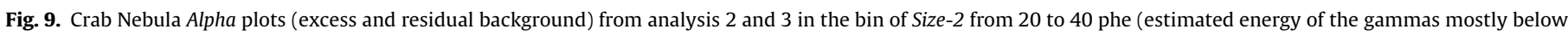
$100 \mathrm{GeV}$ ). Note that in this case we have adjusted the background rate instead of the excess rate to be the same in both cases. The Alpha cut applied is of $12^{\circ}$.

is chosen to minimize the variance of the true energies of the event samples resulting from the split [21], rather than their purity. No background event sample is needed for this kind of training.

The set of image parameters typically used for the estimation of the energy is: Size, Width, Length, Dist, Conc, Leakage and Zenith Angle. In order to evaluate the improvement in the energy reconstruction due to the use of the signal timing, the energy of a test sample of Monte Carlo $\gamma$ events (different from the sample used for the training) was evaluated and compared to the true known energy of the primary gamma-rays. An improvement in the energy reconstruction is expected if the image parameter Time Gradient is added to the default set of parameters since it provides information about the impact parameter of the shower. This should help to avoid the degeneracy between small, nearby showers and the large, distant ones. As introduced in Section 2.3, both Dist and Time Gradient parameters are well correlated with the IP and can be used for its estimation. Notice that the correlation of Dist is rather good for small IP event whereas for larger IP the estimation with Dist becomes poorer (Fig. 3). In case of Time Gradient the correlation is better for higher IP values, very likely because for distant showers the time structure of the images is more pronounced and as a consequence more precisely measured.

The distributions of the quantity $\left(E_{\text {rec }}-E_{\text {true }}\right) / E_{\text {rec }}$ for different energy bins were used to estimate the quality of the energy reconstruction. In Fig. 10 the black triangles refer to the energy estimation with the standard parameter set, that is, data processed with the $10-5$ phe image cleaning without time constraints. The blue squares correspond to an energy reconstruction performed with the standard parameter set and the time image cleaning 6-3 phe (see Section 2.1), while the red circles are obtained from the time-constrained image cleaning and the Time Gradient image parameter being added to the standard set for the energy estimation. The graph represents the value of the RMS of $\left(E_{\text {est }}-E_{\text {true }}\right) / E_{\text {est }}$ with respect to zero instead of the mean value. This quantity is preferred to the simple RMS as an overall estimator of the quality of the energy reconstruction, since it takes into account not only the spread of the distribution, but also a possible bias with respect to zero (see [17] for more details). "Leakage" effects for images located close to the edge of the camera could be important when the energy reconstruction is performed since the number of photons in the part of the image outside the camera is actually not measured. A standard selection cut Leakage $<10 \%$ is applied in this analysis and tests with tighter Leakage cuts revealed no significant changes with respect to the shown results. The use of the Time Gradient image parameter in the energy estimation yields to a relative improvement in energy reconstruction of around $15 \%$.

\section{RMS respect 0}

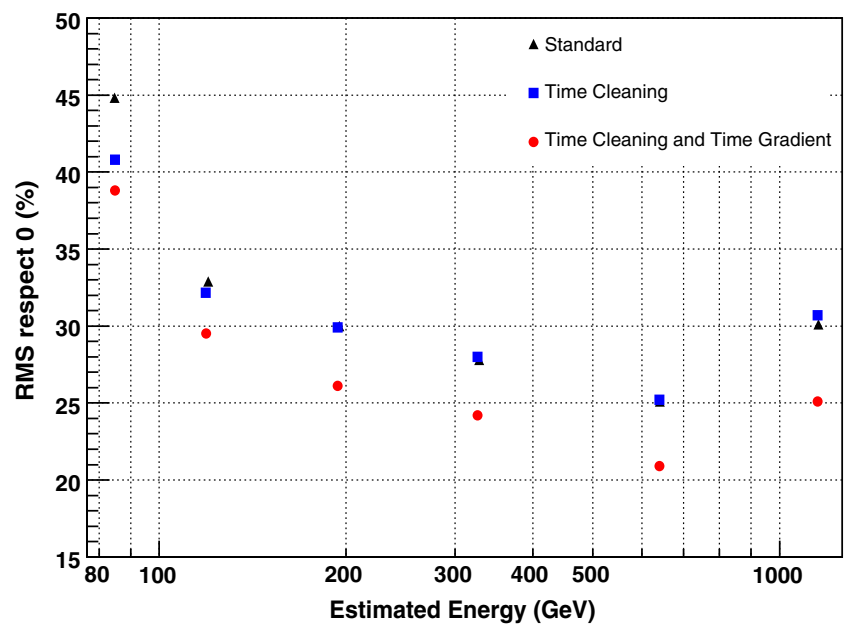

Fig. 10. Graph of the value of the RMS with respect to zero of the quantity $\left(E_{\text {est }}-E_{\text {true }}\right) / E_{\text {est }}$ for different energy bins. This value is an overall estimator of the energy resolution. The black triangle and the blue square show data processed with the standard set of energy estimation parameters (image cleaning 10-5 phe and time image cleaning 6-3 phe, respectively). The red circles correspond to data processed with time image cleaning and also Time Gradient used as energy estimation parameter (time image cleaning 6-3 phe).

\section{Discussion}

We have established that a significant improvement of performance of the MAGIC Cherenkov telescope, both in terms of flux sensitivity and energy resolution, can be achieved by using the timing of signals in the reconstruction of shower images. The reason for the improvement is two-fold: on one hand, the time-constrained image cleaning allows to reduce the cleaning charge levels without adding noise coming mainly from the night sky background light, which results in a lower analysis energy threshold. On the other hand, the timing profile of the images, represented by the Time Gradient, provides information about the shower impact parameter, a relevant quantity which is otherwise poorly determined by a single-dish IACT. The results presented here have been reproduced also by a different analysis within the MAGIC collaboration. This analysis shares the "core" of MARS and is based on [25] but the algorithms for the analysis are developed independently, for example fixed cuts in combinations of the 


\section{SIMULATED GAMMAS}

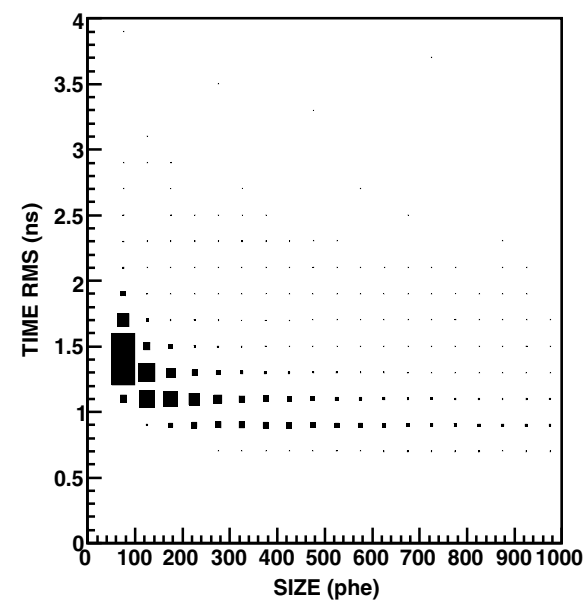

\section{SIMULATED MUONS}

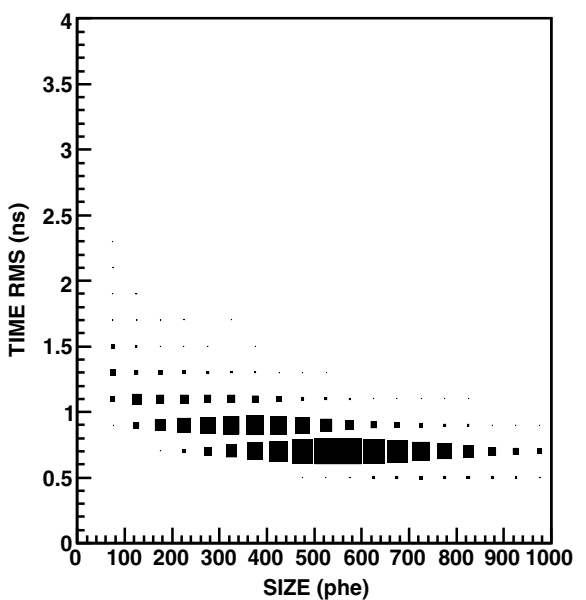

REAL MUONS (arc $>180 \mathrm{deg})$

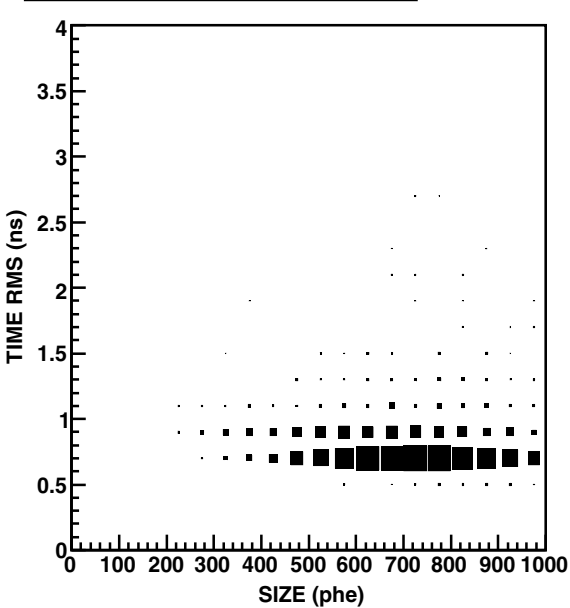

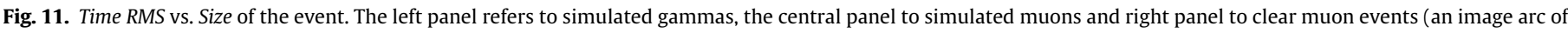

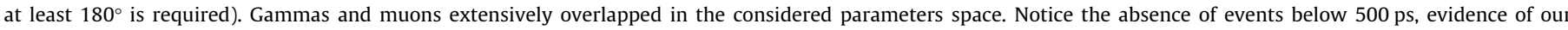

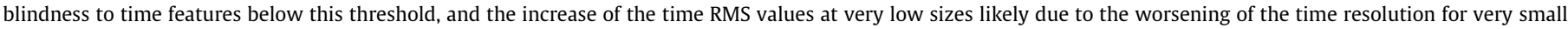
signals.

image parameters are used instead of the Random Forest. Also in this case is the introduction of the Time Gradient led to the described improvement in the background suppression.

From the sensitivity graphs of Section 3.2, it is possible to conclude that the time cleaning alone results in a significant sensitivity improvement in the low energy regime ( $40<$ Size- $2<100$ phe), coming from the increased event statistics. At higher energies it does neither improve nor worsen the telescope performance significantly although the lower cleaning results in more pixels per image. In contrast, the Time Gradient seems to be helpful in the entire energy range accessible to MAGIC. This parameter allows to reject hadron showers whose images are gamma-like in shape and oriented towards the gamma-ray source location on the camera, but whose Dist and Time Gradient parameters are not consistent with what is expected for a gamma shower coming from a point source.

The events with very large Time RMS ( $\gtrsim 1.5 \mathrm{~ns}$ ) are rejected thanks to the Time RMS parameter. The background rejection power shown by the Time RMS in this study is much lower than foreseen in [6]. This is most likely due to the too optimistic assumptions regarding the telescope features made by the authors of that work with respect to the actual characteristics of MAGIC, in particular regarding the reflecting dish. In the final mounting the panels of the MAGIC mirror are staggered in a chessboard pattern to facilitate their movement and to ensure a proper focusing, and this causes the parabolic dish not to be perfectly synchronous. The mirrors staggering together with the other sources of time spread in the acquisition chain, like the jitter in the transit time of the electrons in the PMTs, lead to a time-RMS response larger than expected from the authors of [6]. The value that results from almost synchronous input signals, for example muon events, is $\approx 0.7 \mathrm{~ns}$, a value comparable with the intrinsic time spread of the low energy $\gamma$-events (see Fig. 11). Therefore, the tagging of single distant muons from just their time spread is at the moment not possible.

In conclusion the use of timing information in the analysis of MAGIC data provides a considerably better background suppression and results in an enhancement of about a factor 1.4 of the flux sensitivity to point-like sources, as tested on real observations of the Crab Nebula. This gain is equivalent to doubling the available observation time.

Improvements of the order of $15 \%$ have been found in the event energy reconstruction. In fact the time gradient gives information about the real impact parameter of the shower and therefore it helps to distinguish distant high energy showers from closer, low energy ones.

We expect that this type of timing analysis may also be helpful to future Cherenkov telescopes. Even if the Time Gradient is very likely not useful for stereo IACT systems, this does not exclude that different time-related image parameters can be worth for the reduction of the stereo system data. The time image cleaning algorithm would be instead worth for either stereo and single IACT systems.

\section{Acknowledgements}

We would like to thank the Instituto de Astrofisica de Canarias for the excellent working conditions at the Observatorio del Roque de los Muchachos in La Palma. The support of the German BMBF and MPG, the Italian INFN and Spanish MCINN is gratefully acknowledged. This work was also supported by ETH Research Grant TH 34/043, by the Polish MNiSzW Grant N N203 390834, and by the YIP of the Helmholtz Gemeinschaft.

\section{References}

[1] A.M. Hillas, Cherenkov light images of EAS produced by primary gamma, Proceedings of the XIX International Cosmic Ray Conference 3 (1985) 445448.

[2] A.M. Hillas, The sensitivity of Cherenkov radiation pulses to the longitudinal development of cosmic-ray showers, Journal of Physics G Nuclear Physics 8 (1982) 1475-1492.

[3] M. Hess et al., The time structure of Cherenkov images generated by $\mathrm{TeV}$ gamma-rays and by cosmic rays, Astroparticle Physics 11 (1999) 363-377. eprint arXiv:astro-ph/9812341.

[4] I. De la Calle, S.D. Biller, Extending the sensitivity of air Cherenkov telescopes, Astroparticle Physics 26 (2006) 69-90.

[5] J. Holder, for the VERITAS Collaboration, Exploiting VERITAS timing information, in: Proceedings of the XXIX International Cosmic Ray Conference, vol. 5, 2005, pp. 383-386.

[6] R. Mirzoyan, D. Sobczynska, E. Lorenz, M. Teshima, Tagging single muons and other long-flying relativistic charged particles by ultra-fast timing in air Cherenkov telescopes, Astroparticle Physics 25 (June) (2006) 342-348. eprint arXiv:astro-ph/0605091.

[7] J. Cortina et al., Technical performance of the MAGIC telescope, Proceedings of the XXIX International Cosmic Ray Conference 5 (2005) 359-+.

[8] R. Mirzoyan et al., Ultrafast FADC multiplexer, Nuclear Science 29 (2002) 2473-2476.

[9] F. Goebel, Upgrade of the MAGIC telescope with a multiplexed fiber-optic 2 GSamples/s FADC data acquisition system system, in: Proceedings of the XXX International Cosmic Ray Conference, 2007. 
[10] H. Bartko et al., FADC Pulse Reconstruction Using a Digital Filter for the MAGIC Telescope, 2005, eprint arXiv:astro-ph/0506459.

[11] J. Albert et al., Signal reconstruction for the MAGIC telescope, Nucl. Instr. Meth, A594 (2008) 407, eprint, arxiv:astro-ph/0612385.

[12] M. Gaug, Calibration of the MAGIC Telescope and Observations of Gamma-Ray Burts, Ph.D. Thesis, March 2006, Universitat Autónoma de Barcelona. <http:// magic.mppmu.mpg.de/publications/theses/index.html>.

[13] T. Bretz, R. Wagner, for the MAGIC Collaboration, The MAGIC analysis an reconstruction software, in: Proceedings of the XXIIX International Cosmic Ray Conference, vol. 5, July 2003, p. 2947-+.

[14] J. Albert et al., VHE gamma-ray observation of the Crab Nebula and pulsar with MAGIC, Astrophysical Journal 674 (2008) 1037-1055.

[15] A.N. Otte, Observation of VHE $\gamma$-Rays from the Vicinity of Magnetized Neutron Stars and Development of New Photon-Detectors for Future Ground Based $\gamma$ Ray Detectors, Ph.D. Thesis, Technical University Munich, 2007. <http://nbnresolving.de/urn/resolver.pl?urn:nbn:de:bvb:91-diss-20070924-620881-1$0>$.

[16] D. Tescaro, for the MAGIC Collaboration, Study of the performance and capability of the new ultra-fast 2 GSample/s FADC data acquisition system of the MAGIC telescope, in: Proceedings of the XXX International Cosmic Ray Conference, 2007.

[17] D. Tescaro, Timing Analysis of MAGIC Data After the Installation of the UltraFast 2 GSamples/s FADC Readout, Diploma Thesis, Universitat Autónoma de
Barcelona, 2007. <http://magic.mppmu.mpg.de/publications/theses/ index.htmls.

[18] D. Mazin et al., Model analysis for the MAGIC telescope, in: Proceedings of the XXX International Cosmic Ray Conference, 2007.

[19] M. De Naurois, Analysis methods for atmospheric Cherenkov telescopes, in: Proceedings of the Conference: Towards a Network of Atmospheric Cherenkov Detectors VII, Palaiseau, France, 2005, eprint arXiv:astro-ph/0607247.

[20] V. Fomin et al., New methods of atmospheric Cherenkov imaging for gammaray astronomy. I. The false source method, Astroparticle Physics 2 (1994) 137150.

[21] J. Albert et al., Implementation of the random forest method for the imaging atmospheric Cherenkov telescope MAGIC, Nuclear Instruments and Methods A 588 (2008) 424-432.

[22] T.-P. Li, Y.-Q. Ma, Analysis methods for results in gamma-ray astronomy, Astrophysical Journal 272 (1983) 317-324.

[23] G. Maier, J. Knapp, Cosmic-ray events as background in imaging atmospheric Cherenkov telescopes, Astroparticle Physics 28 (2007) 72-81.

[24] D. Sobczyńska, Natural limits on the $\gamma /$ hadron separation for a stand alone air Cherenkov telescope, Journal of Physics G Nuclear Physics 34 (2007) 22792288.

[25] B. Riegel, T. Bretz, for the MAGIC Collaboration, A systematic study of the interdependence of IACT image parameters, in: Proceedings of the XXIX International Cosmic Ray Conference, vol. 5, 2005, p. 215-+. 